UWThPh-1997-49

HEPHY-PUB $682 / 97$

hep-ph/9712412

\title{
Scalar Top Quark Production at $\boldsymbol{\mu}^{+} \boldsymbol{\mu}^{-}$Colliders
}

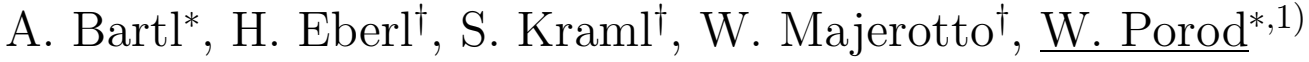 \\ *Institut für Theoretische Physik, Universität Wien, A-1090 Vienna, Austria \\ ${ }^{\dagger}$ Institut für Hochenergiephysik der Österreichischen Akademie der Wissenschaften, \\ A-1050 Vienna, Austria
}

\begin{abstract}
We discuss the production of stops at a $\mu^{+} \mu^{-}$collider. We present numerical predictions for the cross sections within the Minimal Supersymmetric Standard Model. In particular we consider stop production near $\sqrt{s}=m_{H^{0}}$ and $\sqrt{s}=m_{A^{0}}$.
\end{abstract}

\section{Introduction}

The search for supersymmetric particles plays an important rôle at LEP2 and TEVATRON. It will play an even more important rôle at the future colliders LHC, an $e^{+} e^{-}$linear collider with an energy range up to $2 \mathrm{TeV}$, and a $\mu^{+} \mu^{-}$collider with an energy range up to $4 \mathrm{TeV}$. We assume that at the time when a $\mu^{+} \mu^{-}$collider starts operation, supersymmetry will have been discovered at TEVATRON or LHC. While proton colliders are good discovery machines [1, 2, 3], one can do precision measurements at $\mu^{+} \mu^{-}$colliders [国]. Another exciting feature of a $\mu^{+} \mu^{-}$collider is the possibility of producing Higgs bosons in the s-channel [4, 5]. This allows one to measure various Higgs couplings at the Higgs resonances.

In the following our framework is the Minimal Supersymmetric Standard Model (MSSM) [6]. The MSSM implies the existence of five physical Higgs bosons: two scalars $h^{0}, H^{0}$, one pseudoscalar $A^{0}$, and two charged ones $H^{ \pm}$[7]. The top quark has two supersymmetric partners, the lighter stop $\tilde{t}_{1}$ and the heavier stop $\tilde{t}_{2}$. The top quark and the stops give important contributions to Higgs masses due to radiative corrections (see e.g. [8]). Moreover, their contributions to the renormalization group equations can lead to electroweak symmetry breaking when the Higgs parameters evolve from the GUT scale to the electroweak scale [9]. Therefore, the couplings of

${ }^{1}$ Talk presented at the Workshop on Physics at the First Muon Collider and the Front End of a Muon Collider, November 6 - 9, 1997, FNAL, Batavia, Illinois, USA. 
a)
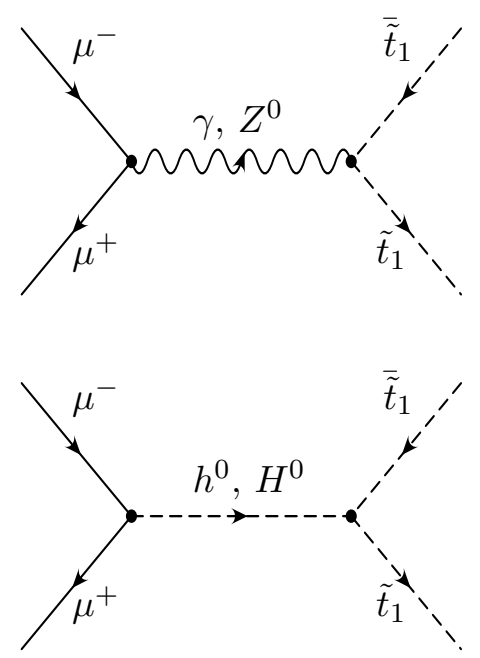

b)
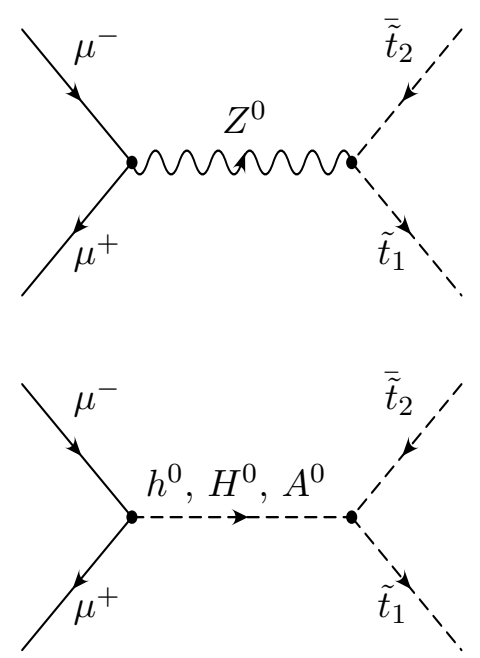

Figure 1: Feynman-graphs for scalar top quark production in $\mu^{+} \mu^{-}$annihilation: a) for $\tilde{t}_{1} \overline{\tilde{t}}_{1}$, b) for $\tilde{t}_{1} \overline{\tilde{t}}_{2}$.

the stops to the neutral Higgs bosons are of special interest. In this contribution we study stop production in $\mu^{+} \mu^{-}$collisions paying particular attention to the energy range near the Higgs resonances.

\section{Production of Stops}

The mass terms of the stops is given by a $2 \times 2$ mass matrix. The diagonal elements are $M_{\tilde{t}_{L}}^{2}=M_{\tilde{Q}}^{2}+m_{Z}^{2} \cos 2 \beta\left(\frac{1}{2}-\frac{2}{3} \sin ^{2} \theta_{W}\right)+m_{t}^{2}$ and $M_{\tilde{t}_{R}}^{2}=M_{\tilde{U}}^{2}+$ $\frac{2}{3} m_{Z}^{2} \cos 2 \beta \sin ^{2} \theta_{W}+m_{t}^{2}$, and the off-diagonal element is given by $m_{t}\left(A_{t}-\mu \cot \beta\right)$. The physical states are characterized by their mass eigenvalues $m_{\tilde{t}_{1}}, m_{\tilde{t}_{2}}$ and the mixing angle $\cos \theta_{\tilde{t}}$.

Figure 1 shows the Feynman-graphs for the processes $\mu^{+} \mu^{-} \rightarrow \tilde{t}_{i} \overline{\tilde{t}}_{j}(i, j=1,2)$. The differential cross section reads

$$
\frac{\mathrm{d} \sigma}{\mathrm{d} \cos \vartheta}=C_{i j}\left(\frac{\kappa_{i j}^{2}}{s^{2}} T_{V V} \sin ^{2} \vartheta+\frac{\kappa_{i j}}{s} T_{V H}^{a} \cos \vartheta+\frac{m_{i}^{2}-m_{j}^{2}}{s} T_{V H}^{b}+T_{H H}\right)
$$

with $s$ the center-of-mass energy squared, $\kappa_{i j}^{2}=\left(s-m_{i}^{2}-m_{j}^{2}\right)^{2}-4 m_{i}^{2} m_{j}^{2}$, and $\vartheta$ the angle between $\mu^{-}$and $\tilde{t}_{i}$. $T_{V V}$ denotes the contribution from $\gamma$ and $Z^{0}$ exchange, $T_{V H}^{a, b}$ the interference terms between gauge and Higgs bosons, and $T_{H H}$ the contribution stemming from the exchange of Higgs bosons. The pure gauge boson contribution, the first part of Equation 1, is the same as for $e^{+} e^{-} \rightarrow \tilde{t}_{i} \tilde{\tilde{t}}_{j}$ and given in [10]. The explicit form of $T_{V H}^{a, b}$ and $T_{H H}$ will be given in a forthcoming paper [11. Notice 


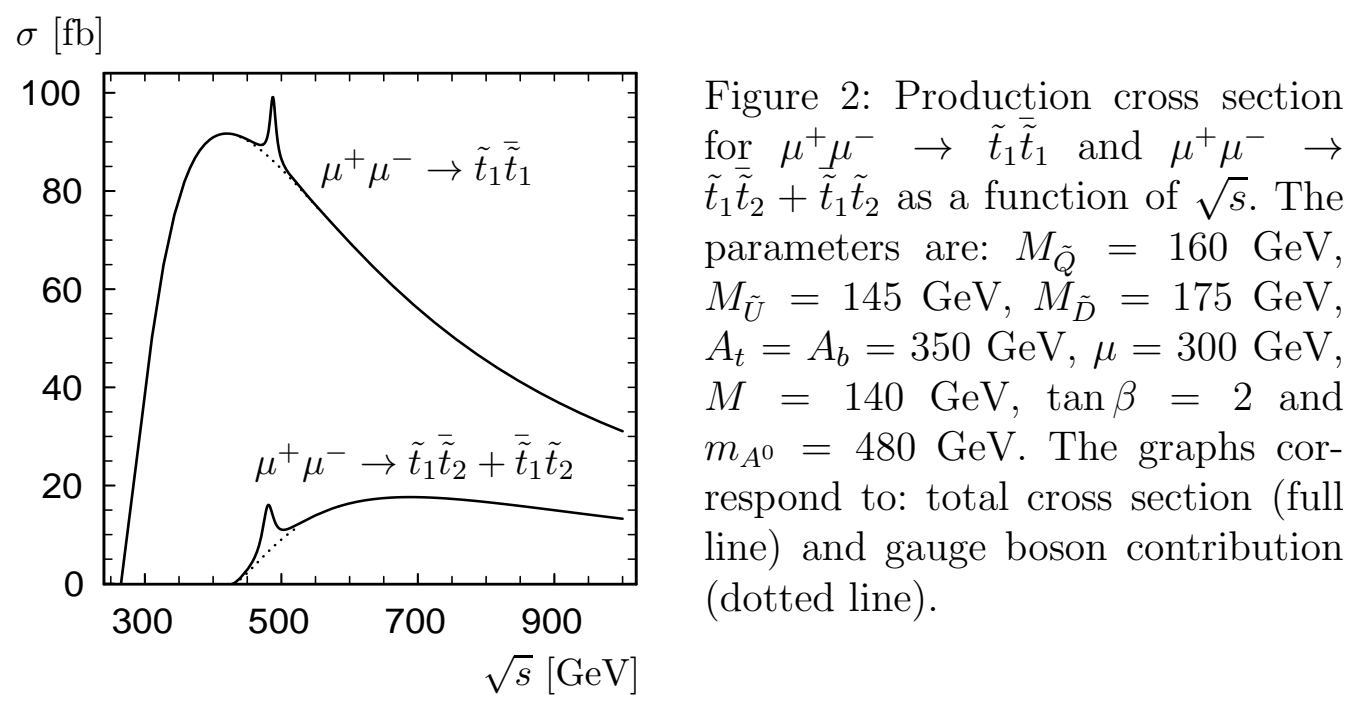

that the gauge boson term has a $\sin ^{2} \vartheta$ dependence whereas $T_{H H}$ and $T_{V H}^{b}$ are independent of $\vartheta$. The $T_{V H}^{a}$ term is proportional to $\cos \vartheta$ giving rise to a forward backward asymmetry. However, this asymmetry is proportional to $m_{\mu}$ and of the order $\lesssim 10^{-4}$ [11]. The following parameters enter the couplings of the stops to the Higgs bosons: $A_{t}, \mu, \cos \theta_{\tilde{t}}, \cos \alpha$, and $\tan \beta$.

In Figure 2 we show the total cross section as a function of $\sqrt{s}$ for $M_{\tilde{Q}}=$ $160 \mathrm{GeV}, M_{\tilde{U}}=145 \mathrm{GeV}, M_{\tilde{D}}=175 \mathrm{GeV}, A_{t}=A_{b}=350 \mathrm{GeV}, \mu=300 \mathrm{GeV}$, $M=140 \mathrm{GeV}, \tan \beta=2$, and $m_{A^{0}}=480 \mathrm{GeV}$. The full lines show the total cross sections and the dotted lines show the gauge boson contributions. The latter ones are identical with the cross sections of $e^{+} e^{-} \rightarrow \tilde{t}_{i} \overline{\tilde{t}}_{j}$. For $\tilde{t}_{1} \overline{\tilde{t}}_{1}$ production the peak results from the $H^{0}$ exchange leading to an enhancement of $\sim 20 \mathrm{fb}$ compared to the gauge boson contribution. For $\tilde{t}_{1} \tilde{t}_{2}$ production the peak is an overlap of the $H^{0}$ and $A^{0}$ resonances because $m_{A^{0}} \simeq m_{H^{0}}$ and the widths of $A^{0}$ and $H^{0}$ are of the order of several $\mathrm{GeV}$ (see e.g. [7, 12, 13]).

In Figure 3 we show the total cross section near the Higgs resonances for various values of $A_{t}$ and the other parameters as above. For $A_{t}=-50(350) \mathrm{GeV}$ one has $m_{\tilde{t}_{1}}=133 \mathrm{GeV}, m_{\tilde{t}_{2}}=296 \mathrm{GeV}$, and $\cos \theta_{\tilde{t}}=0.69(-0.69) . A_{t}=50(250) \mathrm{GeV}$ gives $m_{\tilde{t}_{1}}=187 \mathrm{GeV}, m_{\tilde{t}_{2}}=265 \mathrm{GeV}$, and $\cos \theta_{\tilde{t}}=0.67(-0.67)$. For $A_{t}=150 \mathrm{GeV}$ one gets $m_{\tilde{t}_{1}}=226 \mathrm{GeV}, m_{\tilde{t}_{2}}=233 \mathrm{GeV}$, and $\cos \theta_{\tilde{t}}=0$. The shifts of the peaks are due to radiative corrections to $m_{H^{0}}$. One can clearly see that the widths of the peaks depend on $A_{t}$ and therefore also on the sign of $\cos \theta_{\tilde{t}}$. Note that for $\cos \theta_{\tilde{t}}=0$ the $H^{0} \tilde{t}_{1} \tilde{t}_{1}$ coupling is rather small and, therefore, the peak nearly vanishes. However, at the same time the $A^{0} \tilde{t}_{1} \tilde{t}_{2}$ coupling is large leading to the enhancement and to the shift of the corresponding peak compared to the other $A_{t}$ values. Note that the decay widths of $A^{0}$ and $H^{0}$ into stops are an essential part of the total widths. Therefore, when the peaks are narrower for $\tilde{t}_{1} \overline{\tilde{t}}_{1}$ production then they are broader for $\tilde{t}_{1} \tilde{t}_{2}$ production and vice versa. 

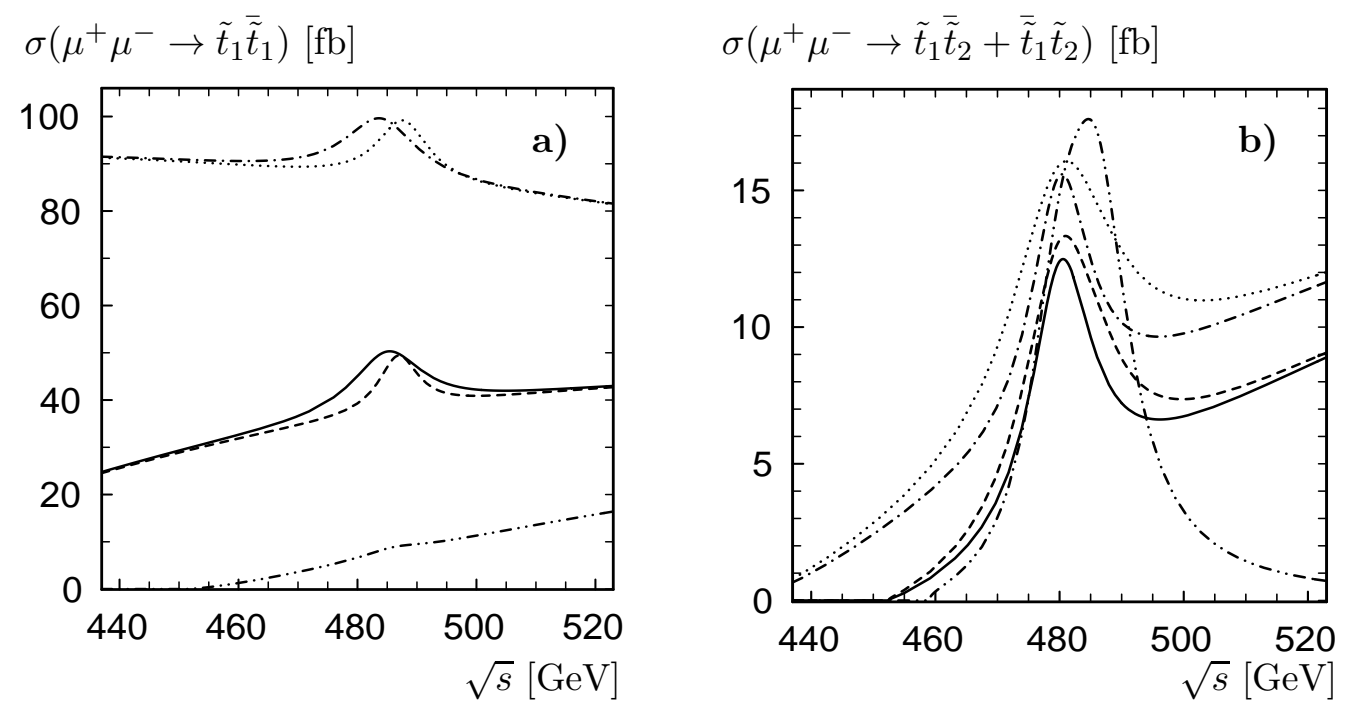

Figure 3: Production cross section (in fb) for a) $\mu^{+} \mu^{-} \rightarrow \tilde{t}_{1} \overline{\tilde{t}}_{1}$ and b) $\mu^{+} \mu^{-} \rightarrow$ $\tilde{t}_{1} \tilde{t}_{2}+\tilde{\tilde{t}}_{1} \tilde{t}_{2}$ as a function of $\sqrt{s}$. The parameters are: $M_{\tilde{Q}}=160 \mathrm{GeV}, M_{\tilde{U}}=$ $145 \mathrm{GeV}, M_{\tilde{D}}=175 \mathrm{GeV}, \mu=300 \mathrm{GeV}, M=140 \mathrm{GeV}, \tan \beta=2$ and $m_{A^{0}}=$ $480 \mathrm{GeV}$. The graphs correspond to $\left(A_{t}=A_{b}\right): A_{t}=-50 \mathrm{GeV}$ (dash-dotted line), $A_{t}=50 \mathrm{GeV}$ (full line), $A_{t}=150 \mathrm{GeV}$ (dotted line), $A_{t}=250 \mathrm{GeV}$ (dashed line) and $A_{t}=350 \mathrm{GeV}$ (dash-dot-dotted line).

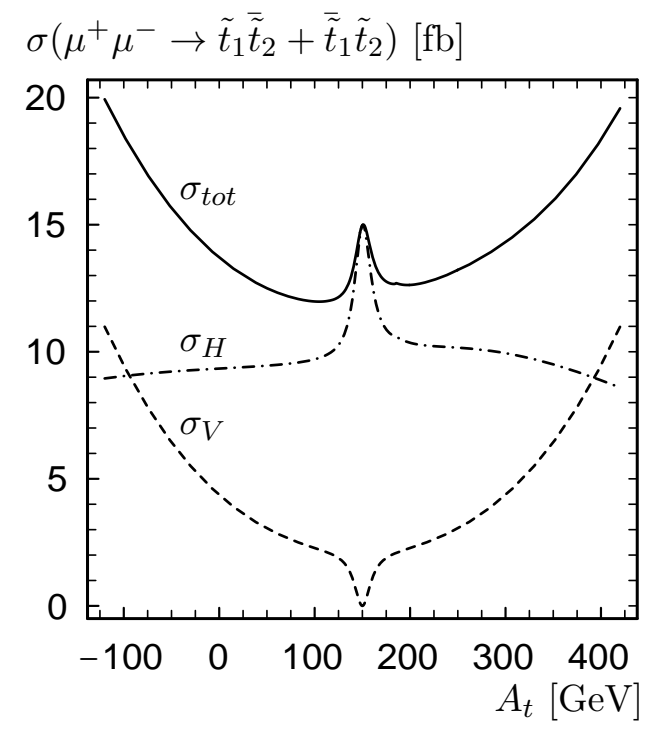

Figure 4: Production cross section (in fb) for $\mu^{+} \mu^{-} \rightarrow \tilde{t}_{1} \overline{\tilde{t}}_{2}+\overline{\tilde{t}}_{1} \tilde{t}_{2}$ as a function of $A_{t}$. The parameters are: $M_{\tilde{Q}}=160 \mathrm{GeV}, M_{\tilde{U}}=145 \mathrm{GeV}$, $M_{\tilde{D}}=175 \mathrm{GeV}, A_{b}=A_{t}, \mu=$ $300 \mathrm{GeV}, M=140 \mathrm{GeV}, \tan \beta=2$ and $\sqrt{s}=m_{A^{0}}=480 \mathrm{GeV}$. The graphs correspond to: total cross section $\sigma_{t o t}$ (full line), Higgs boson contribution $\sigma_{H}$ (dash-dotted line), and gauge boson contribution $\sigma_{V}$ (dashed line).

Figure 1 shows the $A_{t}$ dependence of the cross section $\mu^{+} \mu^{-} \rightarrow \tilde{t}_{1} \overline{\tilde{t}}_{2}+\overline{\tilde{t}}_{1} \tilde{t}_{2}$ for $\sqrt{s}=m_{A^{0}}=480 \mathrm{GeV}$ and the other parameters as above. The Higgs exchange contributes to the total cross section at least $30 \%$. For $\cos \theta_{\tilde{t}}=0\left(A_{t}=150 \mathrm{GeV}\right)$ this contribution reaches $100 \%$. The smaller peak near $A_{t}=200 \mathrm{GeV}$ is due to the 
$H^{0}$ resonance.

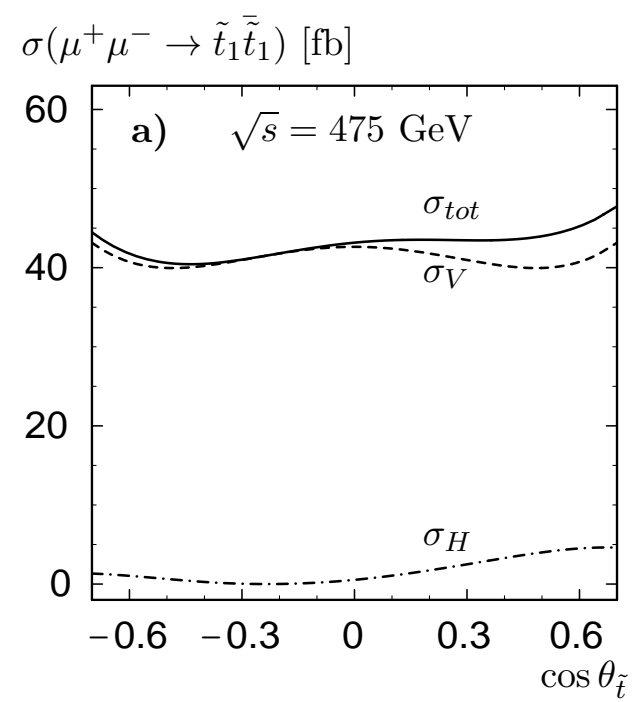

b) $\sigma\left(\mu^{+} \mu^{-} \rightarrow \tilde{t}_{1} \overline{\tilde{t}}_{1}\right)[\mathrm{fb}]$

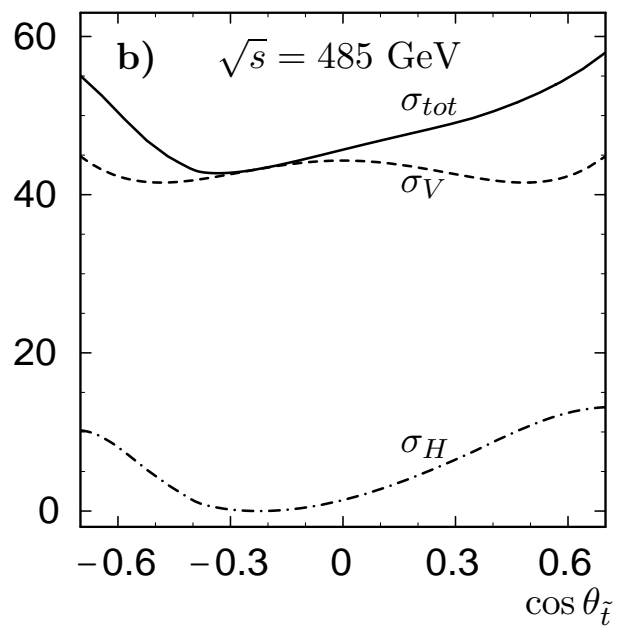

Figure 5: Production cross section for the process $\mu^{+} \mu^{-} \rightarrow \tilde{t}_{1} \overline{\tilde{t}}_{1}$ (in fb) as a function of $\cos \theta_{\tilde{t}}$ for a) $\sqrt{s}=475 \mathrm{GeV}$ and b) $\sqrt{s}=485 \mathrm{GeV}$. The parameters are: $m_{\tilde{t}_{1}}=180 \mathrm{GeV}, M_{\tilde{Q}}=160 \mathrm{GeV}, M_{\tilde{D}}=175 \mathrm{GeV}, \mu=300 \mathrm{GeV}, M=$ $140 \mathrm{GeV}, \tan \beta=2$ and $m_{A^{0}}=480 \mathrm{GeV}$. The graphs correspond to: total cross section $\sigma_{\text {tot }}$ (full line), gauge boson contribution $\sigma_{V}$ (dashed line), and Higgs boson contribution (dash-dot-dotted line).

Figure 5 shows the $\cos \theta_{\tilde{t}}$ dependence of the $\mu^{+} \mu^{-} \rightarrow \tilde{t}_{1} \overline{\tilde{t}}_{1}$ cross section in the energy range close to the $H^{0}$ resonance. The parameters are $m_{\tilde{t}_{1}}=180 \mathrm{GeV}, M_{\tilde{Q}}=$ $160 \mathrm{GeV}, M_{\tilde{D}}=175 \mathrm{GeV}, \mu=300 \mathrm{GeV}, M=140 \mathrm{GeV}, \tan \beta=2$, and $m_{A^{0}}=$ $480 \mathrm{GeV}$. Notice that the Higgs contribution depends on the $\operatorname{sign}$ of $\cos \theta_{\tilde{t}}$.

\section{Conclusions}

We have studied the production of $\tilde{t}_{1} \overline{\tilde{t}}_{1}$ and $\tilde{t}_{1} \overline{\tilde{t}}_{2}$ in $\mu^{+} \mu^{-}$annihilation focusing on the impact of the Higgs resonances in these processes. In particular we have found that one gets important information on the $H^{0} \tilde{t}_{1} \tilde{t}_{1}, H^{0} \tilde{t}_{1} \tilde{t}_{2}$ and $A^{0} \tilde{t}_{1} \tilde{t}_{2}$ couplings.

\section{Acknowledgments}

We are very grateful to M. Carena and S. Protopopescu for their kind invitation to this interesting and inspiring workshop. This work was supported by the "Fonds zur Förderung der wissenschaftlichen Forschung" of Austria, project no. P10843-PHY. 


\section{References}

[1] A. Bartl, J. Soderqvist et al., Proc. of the Workshop on New Directions for High Energy Physics, Snowmass, Colorado, 1996, and references therein.

[2] F. Paige, these Proceedings.

[3] I. Hinchliffe, these Proceedings.

[4] J. Gunion, these Proceedings.

[5] H. E. Haber, these Proceedings.

[6] H. E. Haber and G. L. Kane, Phys. Rep. 117, 75 (1985).

[7] J. F. Gunion, H. E. Haber, G. L. Kane, and S. Dawson, The Higgs Hunter's Guide, Addison-Wesley, 1990, and references therein.

[8] J. Ellis, G. Ridolfi, and F. Zwirner, Phys. Lett. B 257, 83 (1991); Phys. Lett. B 262, 477 (1991).

[9] L. Ibáñez and G. G. Ross, Phys. Lett. B 110, 215 (1982).

[10] H. Eberl, A. Bartl, W. Majerotto, Nucl. Phys. B 472, 481 (1996).

[11] A. Bartl, H. Eberl, S. Kraml, W. Majerotto, and W. Porod, in preparation.

[12] A. Bartl, H. Eberl, K. Hidaka, T. Kon, W. Majerotto, and Y. Yamada, Phys. Lett. B, 402, 303 (1997)

[13] A. Djouadi, J. Kalinowski, P. Ohmann, and P.M. Zerwas, Zeit. f. Phys. C 74, 93 (1997) 